\title{
Exosomes derived from hypoxic epithelial ovarian cancer deliver microRNA-940 to induce macrophage $\mathrm{M} 2$ polarization
}

\author{
XIN CHEN, XIANG YING, XINJING WANG, XIAOLI WU, QINYI ZHU and XIPENG WANG \\ Department of Gynecology, Shanghai First Maternity and Infant Hospital, \\ Affiliated to Tongji University, Jingan, Shanghai 200036, P.R. China
}

Received December 25, 2016; Accepted May 24, 2017

DOI: $10.3892 /$ or.2017.5697

\begin{abstract}
Hypoxia is a common feature of solid tumors. It is closely related to tumor progression. Exosomal microRNAs derived from cancers are considered to be mediators between cancer cells and the tumor microenvironment. In addition, the number of tumor-associated macrophages (TAMs) in the tumor microenvironment has also been demonstrated to correlate with tumor development. However, the relationship between tumor-secreted exosomes and TAM polarization under hypoxic conditions during tumor progression is not clear. Herein, we demonstrated that hypoxia induces the high expression of microRNA-940 (miR-940) in exosomes derived from epithelial ovarian cancer (EOC). We also found that miR-940 is highly expressed in exosomes isolated from ascites of EOC patients. Moreover, the overexpression of miR-940 in macrophages delivered by exosomes stimulated M2 phenotype polarization, while the M2 subtype macrophages promoted EOC proliferation and migration. These results highlight the function of hypoxia in enhancing the high level of expression of miR-940 in tumor exosomes taken up by macrophages. We also showed that the tumor-promoting function of miR-940 is mediated by TAM polarization in EOC. These findings show that tumor-derived exosomal miR-940 induced by hypoxia plays an important role in stimulating TAM polarization in the progression of EOC.
\end{abstract}

Correspondence to: Dr Xipeng Wang, Department of Gynecology, Shanghai First Maternity and Infant Hospital, Affiliated to Tongji University, Room 508, 536 Changle Road, Jingan, Shanghai 200036, P.R. China

E-mail: xipengwang@hotmail.com

Abbreviations: EOC, epithelial ovarian cancer; TAM, tumorassociated macrophage; IL, interleukin; TEXs, tumor-derived exosomes; MDSCs, myeloid-derived suppressor cells; SEM, standard error of the mean; TEM, transmission electron microscopy

Key words: hypoxia, exosomes, miR-940, macrophage polarization, epithelial ovarian cancer

\section{Introduction}

Epithelial ovarian cancer (EOC), because of the late diagnosis and progressive development of drug resistance, remains the most lethal gynecologic cancer (1). Despite diverse clinical treatments that have been put into practice, EOC is still the fifth most common cause of malignant death. Hence, elucidating the molecular and cellular mechanisms of EOC tumorigenesis is essential for effective early diagnosis and target-based therapies.

The tumor microenvironment plays a critical role in cancer initiation, maintenance and progression $(2,3)$. The tumor microenvironment is a complex network. It contains secreted factors, exosomes, immune cells, fibroblasts, and mesenchymal stem cells (3). Tumor development and progression are greatly influenced by the modulation of innate and adaptive immune responses to cancer. Through an inflammatory response, peripheral monocytes are recruited to the tumor (4). Then, the monocytes are reprogrammed by molecular factors in the tumor microenvironment and obtain specific tumor-associated macrophage (TAM) properties (5). Among the infiltrating leukocytes, TAMs have an abundant population (6). TAMs can regulate the tumor microenvironment via initiating fibrosis, suppressing immune detection and promoting angiogenesis (7). According to different polarizations, macrophages can be separated into two phenotypic differentiations: classically activated (M1) and alternatively activated (M2) macrophages (8). M1 macrophages are usually considered pro-inflammatory and cytotoxic due to producing interleukin (IL)-12, tumor necrosis factor- $\alpha$ and reactive oxygen intermediates (9). In contrast, M2 macrophages are anti-inflammatory and promote wound healing through secreting transforming growth factor- $\beta$, IL- 6 and arginase-1 (10). M1 macrophages are generally referred to as tumor suppressive, and M2 macrophages are considered tumor promoting (11). TAMs are mainly constituted by M2 phenotypes (12). In various tumor types, the density of M2 macrophages within tumors is negatively correlated with the survival rate of patients and positively facilitates tumorigenesis (11). Additionally, an ovarian cancer clinical analysis also demonstrated that a high CD163 (the M2 marker) level is associated with poor clinical outcomes (13). Nevertheless, 
the details of the mechanism by which macrophage polarization occurs during cancer progression are poorly understood.

Exosomes are nanometric phospholipid bilayer-enclosed vesicles $(30-100 \mathrm{~nm})$ that can be secreted by nearly all kinds of cells into the extracellular microenvironment. Most recent cancer research has suggested that exosomes are crucial mediators in intercellular communication during tumor progression (14). Secreted exosomes from cancer cells can deliver a broad set of biological contents to nearby or distant recipient cells, including TAMs, T cells, dendritic cells (DCs), fibroblasts and epithelial cells (15). Tumor-derived exosomes (TEXs) carry genetic lipids, proteins, mRNAs, miRNAs, and DNA segments to regulate cancer immunity (16). In ovarian cancer, TEXs can inhibit dendritic cell maturation, promote immunosuppressive myeloid-derived suppressor cell (MDSC) differentiation and suppress tumor-reactive T-cells (17).

Hypoxia is a hallmark of solid tumors. Under hypoxic conditions, tumor cells exhibit increased aggressiveness and dissemination (18-20). In breast cancer, hypoxia can enhance exosome release (21). Berchem and colleagues found that high levels of miR-23a (compared with normoxic tumor cells) in hypoxic tumor-derived microvesicles inhibit natural killer (NK) cell function (22). Moreover, Ling et al demonstrated that there are 108 miRNAs differentially expressed in normoxic and hypoxic oral squamous cell carcinoma-derived exosomes. Furthermore, miR-21, one of the significantly reduced miRNAs, elicited a prometastatic phenotype (23). However, the relationship between hypoxic TEX and TAM polarization in ovarian cancer is unclear. Thus, we hypothesized that TEXs transported from hypoxic ovarian cancer cells to surrounding macrophages can induce M2 phenotype polarization via miRNAs and then enhance ovarian cancer pathology.

\section{Materials and methods}

Ascites samples. With approval by the Ethics Committee of Shanghai First Maternity and Infant Hospital, peritoneal fluids from three patients with benign ovarian diseases and ascites from three pre-therapy EOC patients were collected from Shanghai First Maternity and Infant Hospital, Tongji University (Shanghai, China). All the patients gave informed consent. The total exosomes in peritoneal fluids and ascites were isolated by using ExoQuick exosome precipitation solution reagent according to the manufacturer's instructions (SBI System Biosciences).

Exosome extraction, labeling and tracking. We used the total exosome isolation reagent (from cell culture medium) (Invitrogen, CA, USA) to obtain purified exosomes from exosome-free culture medium of SKOV3. After centrifuging the medium at 2,500 $\mathrm{rpm}$ for $30 \mathrm{~min}$, we retrieved the cell culture supernatant and mixed it with the total exosome isolation reagent. Then, after incubation at $4^{\circ} \mathrm{C}$ overnight, exosomes were centrifuged at $10,000 \times \mathrm{g}$ for $1 \mathrm{~h}$ at $4^{\circ} \mathrm{C}$ and labeled with D384 (Invitrogen), a phospholipid membrane dye (red stain). Labeled exosomes were then added to DAPI-stained (blue stain) macrophages. After incubation at $37^{\circ} \mathrm{C}$ for $2 \mathrm{~h}$, macrophages were observed using a Leica DM-LB confocal microscope.
Electron microscopy. Exosome pellets resuspended in PBS buffer were dropped onto a carbon-coated copper electron microscope grid, as described previously (24). We used a J Tecnai G2 F20 ST transmission electron microscope to observe the exosomes from cell culture medium, peritoneal fluids and ascites.

Cell culture and hypoxia treatment. The human EOC cell line SKOV3 and the monocyte cell line U937 were obtained from Fuheng Bio (Shanghai, China). Cells were cultured using RPMI-1640 medium (Invitrogen) with 10\% FBS (Invitrogen). The cell culture medium was ultracentrifuged at $100,000 \mathrm{x} \mathrm{g}$ for $20 \mathrm{~h}$ to obtain exosome-free medium. To simulate the hypoxic growing conditions of a solid tumor, SKOV3 cells were cultured under $1 \% \mathrm{O}_{2}$ (hypoxic) conditions, balanced with $\mathrm{N}_{2}$ in an $\mathrm{O}_{2} / \mathrm{CO}_{2}$ incubator (Sanyo). The control group was treated in $20 \% \mathrm{O}_{2}$ (normoxic) conditions. U937 cells $\left(1 \times 10^{6}\right)$ were exposed to $100 \mathrm{ng} / \mathrm{ml}$ PMA (Sigma-Aldrich, St. Louis, MO, USA) for $24 \mathrm{~h}$ to induce U937 differentiation into macrophages.

miRNA transfection. Macrophages were seeded on $60-\mathrm{mm}$ dishes and were transfected with miR-negative control and miR-940 mimic (GenePharma, Shanghai, China) using HiperFect transfection reagent according to the manufacturer's instructions (Qiagen GmbH, Hilden, Germany). After 48 h, transfected macrophages were washed with PBS, and the cell culture media of macrophages were harvested 3 days later.

$R N A$ preparation and $q R T-P C R$. Total RNAs from exosomes were extracted using RA808A-1 (SBI System Biosciences) according to the manufacturer's instructions, and TRIzol (Invitrogen) was used to extract cellular RNAs. We used miScript Reverse Transcription kit to reverse-transcribe miRNA to cDNA and analyzed quantitative PCR of miRNAs using miScript SYBR Green PCR kit, according to the manufacturer's instructions (Qiagen $\mathrm{GmbH}$ ). Then, we used the $2^{-\triangle \Delta C T}$ method to calculate gene expression. U6 was used to normalize the miRNA results. The primer sequences are as follows: miR-940, 5'-AAGGCAGGGCCCCCGCTCCCC-3'; U6, 5'-CAAGGATGACACGCAAATTCG-3'.

Western blot analysis. Total proteins lysed from exosomes and macrophages, treated with normoxic and hypoxic exosomes $(100 \mu \mathrm{g} / \mathrm{ml})$ or miR-negative control and miR-940 mimic for 48-96 h, were separated on a 10\% SDS PAGE gel. The gels were then transferred to polyvinylidenedifluoride membranes (Millipore). After blocking with 7\% non-fat milk for $2 \mathrm{~h}$, membranes were incubated with rabbit anti-CD63 (1:1,000, Santa Cruz Biotechnology), mouse anti-CD81 (1:1,000, Santa Cruz Biotechnology), rabbit anti-GAPDH (1:1,000, Cell Signaling Technology), mouse anti-CD163 (1:200, Santa Cruz Biotechnology) or mouse anti-CD206 (1:100, Santa Cruz Biotechnology) at $4^{\circ} \mathrm{C}$ overnight. Anti-mouse or anti-rabbit IgG (1:2,000, Cell Signaling Technology) was used as the secondary antibody.

Migration assay. For the migration assay, $1.5 \times 10^{5}$ SKOV3 cells were seeded in the top chamber of Transwell chambers (Corning Inc., USA) with $8-\mu \mathrm{m}$ inserts. The cell culture 
A

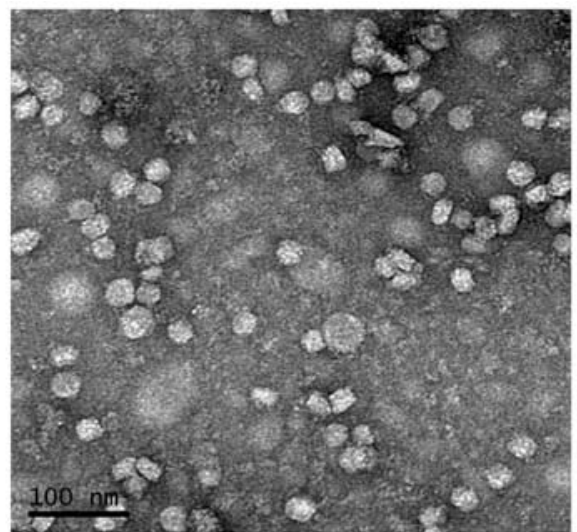

B

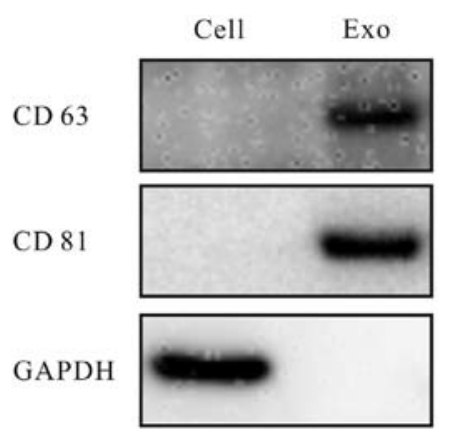

C
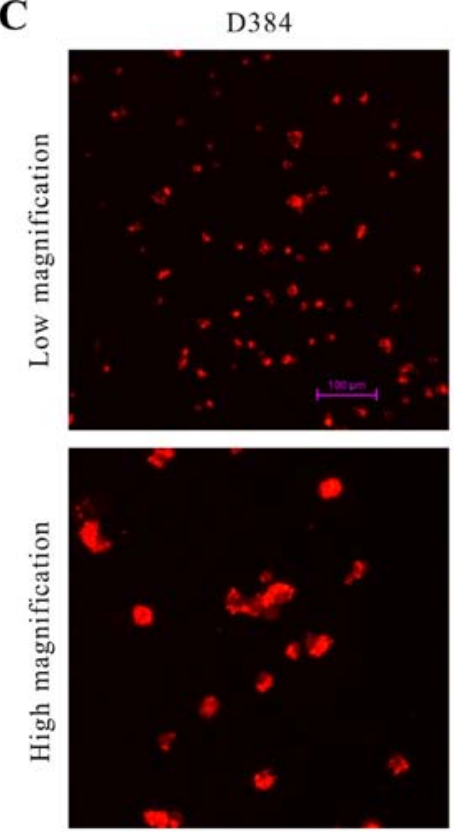
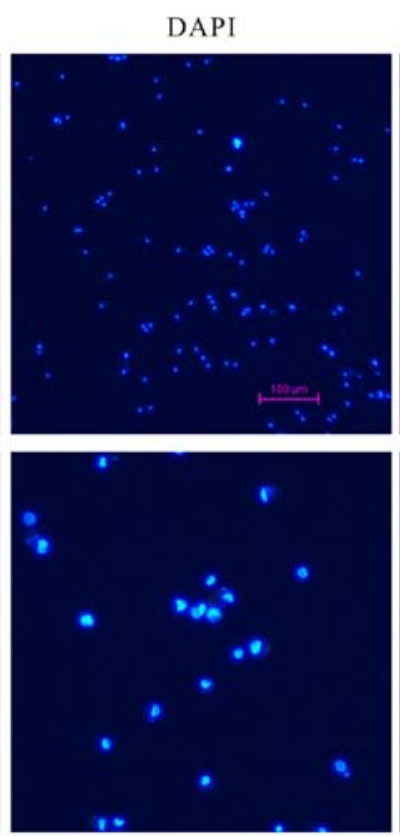
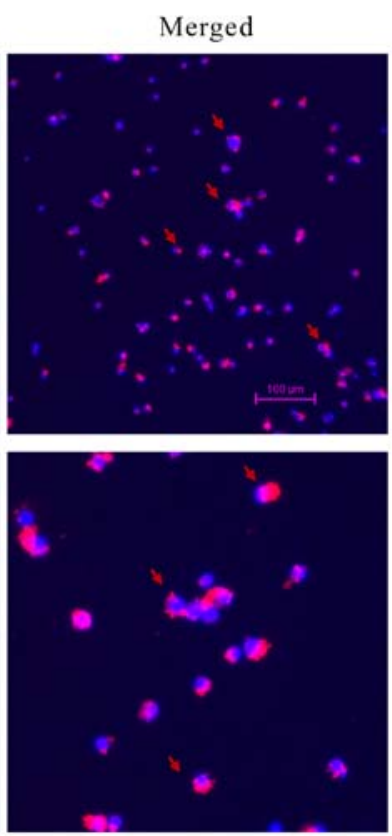

Figure 1. Identification of exosomes secreted by SKOV3 cells. Exosomes were internalized by macrophages. (A) Representative electron micrograph of exosomes isolated from SKOV3-conditioned medium reveals the typical morphology and size (30-80 nm). (B) Western blot analysis shows the presence of exosome markers CD63 and CD81 and the absence of GAPDH in exosomes (exo) derived from the conditioned medium of SKOV3 cells. (C) Representative fluorescence microscopy images show the internalization of D384-labeled exosomes (red) by DAPI-labeled macrophages (blue), as shown by the red arrows.

media from miR-940 mimic-transfected macrophages or miR-negative control-transfected macrophages were added in the bottom chamber. After 48-72 h of incubation, cells that migrated through the membrane were fixed with methanol and stained with crystal violet $1 \%$. Cells were counted in 4 random fields.

MTS proliferation assay. SKOV3 cells were seeded in 96-well plates $\left(2 \times 10^{3}\right.$ cells/well) and cultured with $50 \mu 1$ miR-940-transfected macrophage culture medium mixed with $50 \mu \mathrm{l}$ ordinary medium for $48 \mathrm{~h}$. Cells cultured with $50 \mu \mathrm{l}$ miR-negative control-transfected macrophage culture medium and $50 \mu \mathrm{l}$ ordinary medium were also seeded as a negative control. MTS solution reagent $(20 \mu \mathrm{l})$ (Promega Biosciences, CA, USA) was added to each well. After $2 \mathrm{~h}$ at $37^{\circ} \mathrm{C}$ with $5 \% \mathrm{CO}_{2}$ in incubator, cell proliferation was measured through a $96-$-well plate reader.

Statistical analysis. Data are presented as the mean \pm standard error of the mean (SEM) for at least 3 independent experiments. Student's t-test or the Mann-Whitney U test was used to analyze the significant differences between groups. SPSS 19.0 was applied to perform statistical analyses. $\mathrm{p}<0.05$ was considered statistically significant.

\section{Results}

Identification of exosomes secreted by EOC cells and exosomes taken by macrophages. Exosomes derived from SKOV3 cells in the cell culture supernatant were initially purified by using a total exosome isolation reagent. The extracted exosomes were examined using transmission electron microscopy (TEM), which revealed that the specific exosome morphology and size ranged from 30 to $80 \mathrm{~nm}$ in diameter (Fig. 1A). The known exosome markers CD63 and CD81 were further analyzed by western blotting (Fig. 1B). Abundant biologic information was packaged into the exosomes and internalized by target cells (25). We also found that the TEXs were taken up by macrophages. Exosomes were labeled with D384 (red stain), and DAPI (blue stain) was used to show the nuclear structure 
A

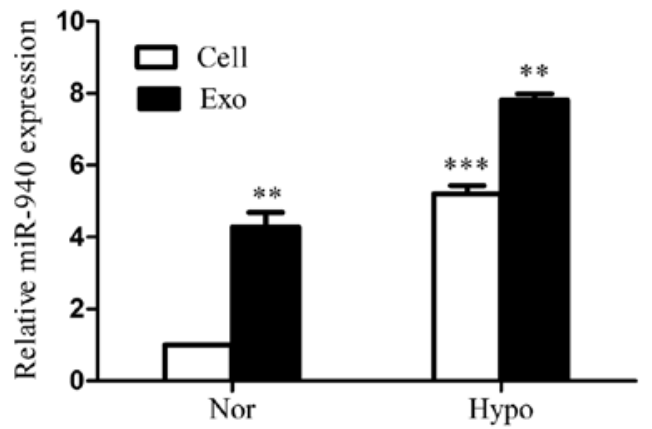

B

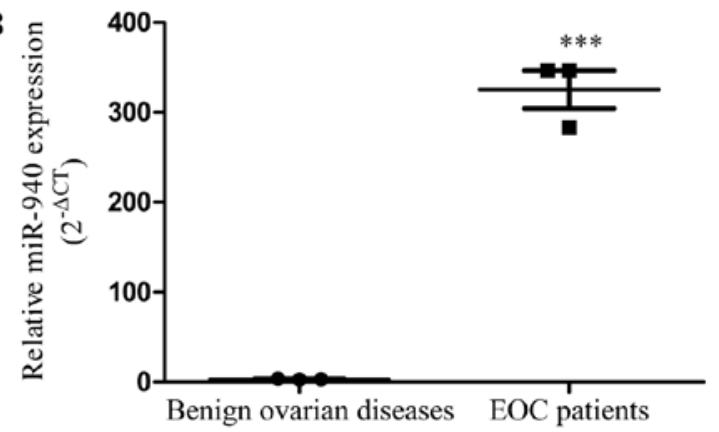

Figure 2. Hypoxia induces expression of miR-940 in SKOV3-derived exosomes, and miR-940 was highly expressed in the exosomes from ascites of EOC patients. (A) SKOV3 cells were co-cultured under normoxic (nor) or hypoxic (hypo) conditions, and the miR-940 expression levels in the cells and corresponding exosomes were measured by qRT-PCR. ${ }^{* *} \mathrm{p}<0.01,{ }^{* * *} \mathrm{p}<0.001$, compared with normoxic cells. (B) MicroRNAs were extracted from the exosomes from ascites of three EOC patients and peritoneal fluids from three patients with benign ovarian diseases. miR-940 expression was detected by qRT-PCR. ${ }^{* * *}$ p $<0.001$, compared with benign ovarian diseases.

A

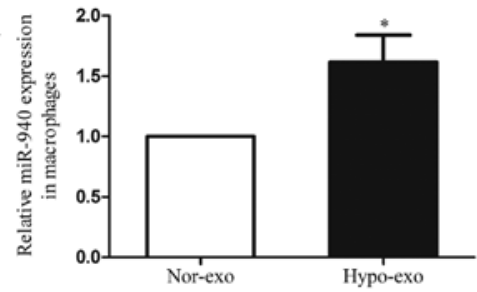

C

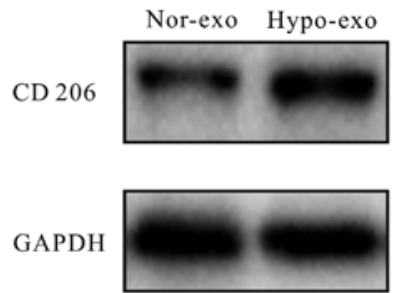

$\mathbf{E}$

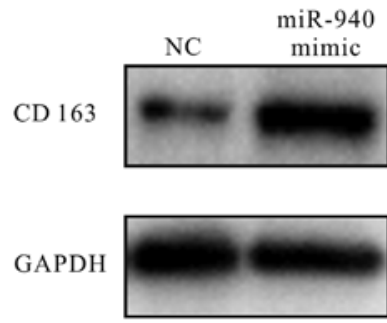

B

Nor-exo Hypo-exo

CD 163

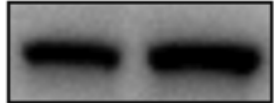

GAPDH

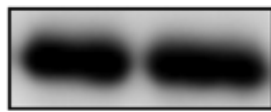

D

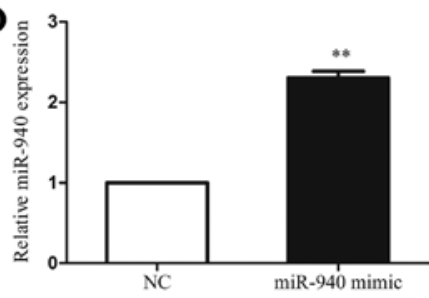

$\mathbf{F}$

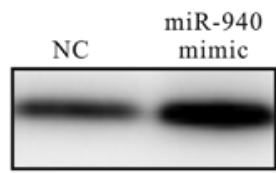

GAPDH

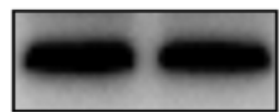

Figure 3. Increased expression of miR-940 in unpolarized macrophages drove macrophages toward a TAM-like phenotype. Unpolarized macrophages were treated with normoxic SKOV3-derived exosomes (nor-exo) or hypoxic SKOV3-derived exosomes (hypo-exo) (100 $\mu \mathrm{g} / \mathrm{ml})$. (A) After 3 days, miR-940 expression levels in macrophages were detected by qRT-PCR. "p<0.05, compared with macrophages with normoxic SKOV3-derived exosomes. (B and C) The M2-phenotype markers CD163 and CD206 of macrophages were analyzed by western blot analysis. Macrophages were transfected with the miR-negative control (NC) or the miR-940 mimic (D). The miR-940 was tested by qRT-PCR. ${ }^{* *}$ p<0.01, compared with NC. (E and F) After 48 h of transfection, the expressions of CD163 and CD206 of macrophages were detected by immunoblotting.

of macrophages. Fluorescence microscopy was used to show that exosomes were located in the cytoplasm of macrophages (Fig. 1C).

Hypoxia induces overexpression of miR-940 both in EOC cells and EOC-derived exosomes, and exosomes isolated from ascites of EOC patients have high expression levels of $m i R-940$. miRNAs in exosomes have been shown to play an important role in tumor progression (16). The expression levels of miRNAs vary by cell type and condition. Thus, in referring to the literature, we found that the overexpressions of miR-940 in pancreatic carcinoma (26) and gastric cancer (27) were correlated with tumor progression. However, the role of miR-940 in ovarian cancer is unknown. We hypothesized that miR-940 may play an important role in macrophage polarization in EOC. To confirm this hypothesis, we first studied the relationship between miR-940 and hypoxia.

The SKOV3 cells were cultured under $20 \% \mathrm{O}_{2}$ (normoxia) or $1 \% \mathrm{O}_{2}$ (hypoxia). Exosomes were isolated, and total miRNA was extracted after $72 \mathrm{~h}$. The qRT-PCR results show that the 


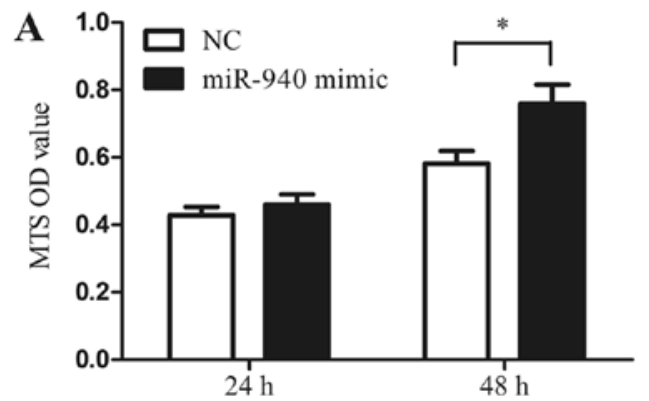

B
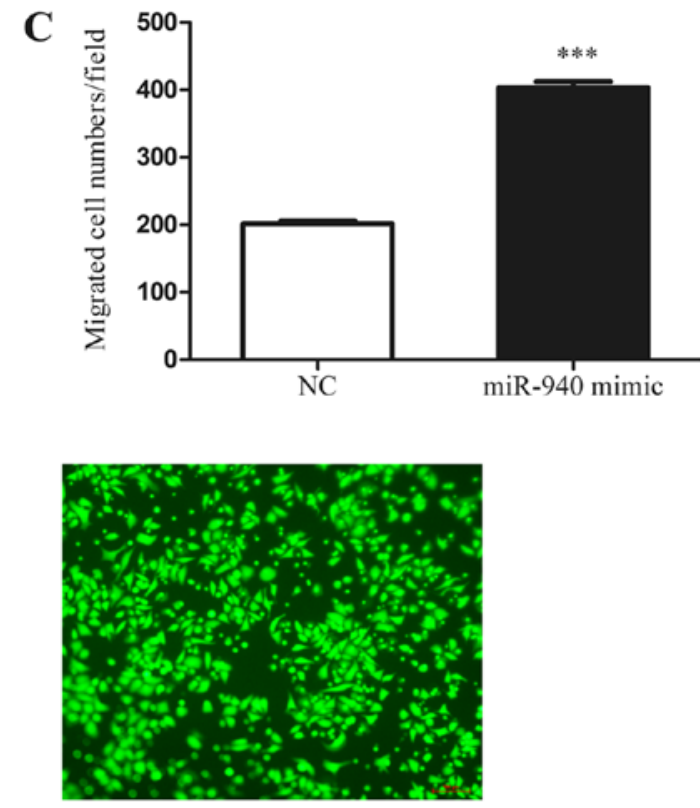

$\operatorname{miR}-940 \mathrm{mimic}$

Figure 4. miR-940-induced M2-phenotype macrophages promote the proliferation and migration of EOC cells. SKOV3 cells were incubated with conditioned medium of miR-negative control (NC-transfected macrophages) or the miR-940 mimic-transfected macrophages. (A) Proliferation capacity of SKOV3 cells was assessed by using the MTS proliferation assay. Cell viability was determined by OD value. * $<<0.05$, compared with NC. (B and C) Transwell assay was used to analyze the migration capacity of SKOV3 cells, and migrated cells were counted by using ImageJ. ${ }^{* * *}$ p $<0.001$, compared with NC.

expression of miR-940 is significantly higher in hypoxic cells and exosomes compared with in the normoxic group (Fig. 2A). Additionally, we explored the degree of miR-940 expression in exosomes isolated from ascites of EOC patients $(n=3)$. Compared with patients with benign ovarian diseases, miR-940 had a higher expression level in exosomes isolated from ascites of the EOC patients (Fig. 2B).

Increased miR-940 expression in macrophages induces M2 polarization. To investigate the function of hypoxic exosomes carrying abundant miR-940 in the tumor microenvironment, we co-cultured the unpolarized macrophages with normoxic exosomes or hypoxic exosomes isolated from SKOV3 cells. After 3 days, we analyzed the miRNA expression of macrophages and found that the miR-940 expression was much higher in the hypoxic exosome-educated macrophages than in the normoxic group (Fig. 3A). Moreover, compared with the normoxic exosome macrophages, the hypoxic exosome macrophages had higher expression levels of the M2-type markers CD163 and CD206 (Fig. 3B and C). Then, to evaluate the relationship between the M2 polarization of macrophages and the increased miR-940 expression in hypoxic exosomes, we transfected unpolarized macrophages with either the miR-negative control or miR-940 mimic (Fig. 3D). After transfection, high expression of miR-940 in macrophages enhanced the CD163 and CD206 protein expressions remarkably (Fig. 3E and F).

miR-940-induced M2 macrophages promote EOC cell proliferation and migration. In addition, to further investigate whether the miR-940-induced M2-phenotype macrophages have the characteristic function of tumor promotion, we collected the conditioned medium of miR-940 mimic-transfected macrophages. Normoxic SKOV3 cells were treated with the conditioned medium, and the MTS proliferation assay was performed. As shown in the results, the conditioned medium from miR-940-induced M2-phenotype macrophages significantly promoted the proliferation of SKOV3 cells (Fig. 4A). Furthermore, the Transwell assay also indicated that miR-940-induced M2-phenotype macrophages promote SKOV3 cell migration (Fig. 4B and C).

\section{Discussion}

In the present study, we showed that the exosomes secreted by EOC cells under hypoxic conditions in vitro have a higher expression of miR-940. After co-culturing the hypoxic exosomes with unpolarized macrophages, we found that the highly expressed exosomal miR-940 activates macrophages to a TAM-like phenotype. Then, we collected the conditioned medium of activated TAM-like macrophages and demonstrated that the conditioned medium could reversely promote EOC cell proliferation and migration. To our knowledge, this is the first study to suggest that exosomes function as a bridge between hypoxic EOC cells and macrophages in the tumor microenvironment by delivering miR-940.

Tumor growth has increasingly been considered a complex system that includes endothelial cells, fibroblasts, myeloid-derived suppressor cells and macrophages (15). The interaction between cancer cells and TAMs, one of the most abundant immune-related stromal cells, plays a crucial role in cancer development (28). The exchange of contents between cells mediated by cell-derived exosomes functions as a bridge between cancer cells and macrophages. To demonstrate whether or not EOC cells can induce macrophage polarization through secreting exosomes, we initially demonstrated the specificity of isolated exosomes (Fig. 1A and B). In Fig. 1C, 
we show that the EOC-derived exosomes could be taken by macrophages. This indicates that, by secreting exosomes, EOC cells may participate in regulating the biologic functions of macrophages that are recruited to the tumor microenvironment.

Recent studies have found that hypoxia is involved in the malignant evolution of cancers. In breast cancer, hypoxia has been postulated to promote exosome release (21), while in ovarian cancer, hypoxia increases tunneling nanotube formation, which stimulates drug resistance (29). Hypoxia induces macrophage polarization in glioblastoma and lung cancer (30,31). However, the role of hypoxia in exosomes and TAM-like macrophage polarization in epithelial ovarian cancer is not clear. We analyzed the expression of miRNAs under normoxic and hypoxic conditions. We found that SKOV3 cells under hypoxic conditions express much more miR-940 than the cells under normoxic conditions, as well as in exosomes (Fig. 2A). As the presence of ascites has been directly associated with peritoneal metastasis and poor prognosis of EOC patients (32), we also isolated exosomes from ascites of EOC patients and demonstrated that miR-940 has a much higher expression level compared with exosomes of peritoneal fluids from patients with benign ovarian diseases (Fig. 2B). These results imply that the increased expression of miR-940 in exosomes induced by hypoxia may play an important role in EOC progression.

It has been generally recognized that exosomes participate in tumor progression by regulating the immune function of immune cells in the tumor microenvironment (14-17). A recent study of melanomas found that tumor-secreted exosomes suppress the proliferation of $\mathrm{T}$ cells (33). Another study on gastric cancer suggested that cancer cell-derived exosomes activate macrophages to increase pro-inflammatory factor expression, which promotes cancer progression (34). Our previous study also demonstrated that EOC-secreted exosomal miR-222-3p induces TAM polarization (24). However, the aberrant expression of exosomal miR-940 induced by hypoxia during macrophage polarization is not well understood. Thus, in order to investigate the function of hypoxic exosomes in macrophage polarization, we co-cultured the unpolarized macrophages with normoxic exosomes or hypoxic exosomes. We found that miR-940 is greatly increased in macrophages compared with the normoxic group (Fig. 3A). Moreover, hypoxic exosomes induced macrophages to express higher levels of the M2-type markers CD163 and CD206 compared to normoxic exosomes. These data suggested that exosomes could deliver miR-940 to macrophages and that hypoxic exosomes could induce macrophages to an M2-like phenotype. The overexpression of $\mathrm{miR}-940$ has been found to promote tumor progression both in pancreatic carcinoma and gastric cancer $(26,27)$. However, to our knowledge, the expression level and biologic function of miR-940 in EOC have not been reported yet. In this study, we showed that miR-940 is aberrantly expressed in the exosomes from ascites of EOC patients. Furthermore, induced by hypoxia, the expression of miR940 in SKOV3 cells and exosomes is increased, while miR-940 could also be transported to macrophages. Based on these findings, we assume that highly expressed miR-940 may play an important role in the process of TAM-like macrophage polarization, which is enhanced by hypoxic exosomes of SKOV3 cells. We transfected unpolarized macrophages with an miR-negative control or miR-940 mimic. After $48 \mathrm{~h}$, the high expression of miR-940 in macrophages enhanced the CD163 and CD206 protein expression remarkably (Fig. 3E and F). These findings suggest that overexpressed exosomal miR-940 promotes M2 macrophage polarization.

Many studies have demonstrated that the tumor promoting function of M2-phenotype macrophages is mediated by soluble factors $(10,11,35)$. Here, to further confirmed that the miR-940-induced M2-phenotype macrophages have a tumor promoting effect, we co-cultured normoxic SKOV3 cells with miR-940-induced M2 macrophage conditioned medium, and found that the proliferation and migration abilities of SKOV3 cells were increased compared with the miR-negative control (Fig. 4).

In conclusion, these data suggest that hypoxia increases the miR-940 levels in EOC-derived exosomes. These miR-940-rich tumor-secreted exosomes, which are internalized by unpolarized macrophages, drive macrophages toward a TAM-like phenotype. These observations demonstrate that exosomes derived from tumor cells may function as messengers that transport miR-940 between hypoxic tumor cells and macrophages, and thus remodel the tumor immune microenvironment of EOC. These findings may provide a theoretical foundation for a new treatment of EOC through the modulation of TAMs.

\section{Acknowledgements}

This study was supported by grants from the National Natural Science Foundation of China (no. 81372787), the Shanghai Municipal Bureau of Health (no. 20134033), the Shanghai Health System Joint Research Project (no. 2013ZYJB0201) and the Top 100 Medical Elite in Shanghai (no. XBR2011065).

\section{References}

1. Siegel RL, Miller KD and Jemal A: Cancer statistics, 2015. CA Cancer J Clin 65: 5-29, 2015.

2. Casey SC, Li Y, Fan AC and Felsher DW: Oncogene withdrawal engages the immune system to induce sustained cancer regression. J Immunother Cancer 2: 24, 2014.

3. Kenny PA, Lee GY and Bissell MJ: Targeting the tumor microenvironment. Front Biosci 12: 3468-3474, 2007.

4. Liu Y and Cao X: The origin and function of tumor-associated macrophages. Cell Mol Immunol 12: 1-4, 2015.

5. Ostuni R, Kratochvill F, Murray PJ and Natoli G: Macrophages and cancer: From mechanisms to therapeutic implications. Trends Immunol 36: 229-239, 2015.

6. Ruffell B, Au A, Rugo HS, Esserman LJ, Hwang ES and Coussens LM: Leukocyte composition of human breast cancer. Proc Natl Acad Sci USA 109: 2796-2801, 2012.

7. Kerkar SP and Restifo NP: Cellular constituents of immune escape within the tumor microenvironment. Cancer Res 72: 3125-3130, 2012.

8. Lopes RL, Borges TJ, Zanin RF and Bonorino C: IL-10 is required for polarization of macrophages to M2-like phenotype by mycobacterial DnaK (heat shock protein 70 ). Cytokine 85: 123-129, 2016.

9. Ribatti D: Mast cells and macrophages exert beneficial and detrimental effects on tumor progression and angiogenesis. Immunol Lett 152: 83-88, 2013.

10. Pal R, Chakraborty B, Nath A, Singh LM, Ali M, Rahman DS, Ghosh SK, Basu A, Bhattacharya S, Baral R, et al: Noble metal nanoparticle-induced oxidative stress modulates tumor associated macrophages (TAMs) from an M2 to M1 phenotype: An in vitro approach. Int Immunopharmacol 38: 332-341, 2016.

11. Lewis CE and Pollard JW: Distinct role of macrophages in different tumor microenvironments. Cancer Res 66: 605-612, 2006. 
12. Mantovani A and Sica A: Macrophages, innate immunity and cancer: Balance, tolerance, and diversity. Curr Opin Immunol 22: 231-237, 2010.

13. Reinartz S, Schumann T, Finkernagel F, Wortmann A, Jansen JM, Meissner W, Krause M, Schwörer AM, Wagner U, Müller-Brüsselbach S, et al: Mixed-polarization phenotype of ascites-associated macrophages in human ovarian carcinoma: Correlation of CD163 expression, cytokine levels and early relapse. Int J Cancer 134: 32-42, 2014.

14. Baroni S, Romero-Cordoba S, Plantamura I, Dugo M, D'Ippolito E, Cataldo A, Cosentino G, Angeloni V, Rossini A, Daidone MG, et al: Exosome-mediated delivery of miR-9 induces cancer-associated fibroblast-like properties in human breast fibroblasts. Cell Death Dis 7: e2312, 2016.

15. Luo Z, Wang Q, Lau WB, Lau B, Xu L, Zhao L, Yang H, Feng M, Xuan Y, Yang Y, et al: Tumor microenvironment: The culprit for ovarian cancer metastasis? Cancer Lett 377: 174-182, 2016.

16. Saleem SN and Abdel-Mageed AB: Tumor-derived exosomes in oncogenic reprogramming and cancer progression. Cell Mol Life Sci 72: 1-10, 2015 .

17. Nawaz M, Fatima F, Nazarenko I, Ekström K, Murtaza I, Anees M, Sultan A, Neder L, Camussi G, Valadi H, et al: Extracellular vesicles in ovarian cancer: Applications to tumor biology, immunotherapy and biomarker discovery. Expert Rev Proteomics 13: 395-409, 2016.

18. Krtolica A and Ludlow JW: Hypoxia arrests ovarian carcinoma cell cycle progression, but invasion is unaffected. Cancer Res 56: 1168-1173, 1996

19. Krishnamachary B, Berg-Dixon S, Kelly B, Agani F, Feldser D, Ferreira G, Iyer N, LaRusch J, Pak B, Taghavi P, et al: Regulation of colon carcinoma cell invasion by hypoxia-inducible factor 1 . Cancer Res 63: 1138-1143, 2003.

20. Yoon SO, Shin S and Mercurio AM: Hypoxia stimulates carcinoma invasion by stabilizing microtubules and promoting the Rab11 trafficking of the alpha6beta4 integrin. Cancer Res 65: 2761-2769, 2005.

21. King HW, Michael MZ and Gleadle JM: Hypoxic enhancement of exosome release by breast cancer cells. BMC Cancer 12: 421, 2012.

22. Berchem G, Noman MZ, Bosseler M, Paggetti J, Baconnais S, Le Cam E, Nanbakhsh A, Moussay E, Mami-Chouaib F, Janji B, et al: Hypoxic tumor-derived microvesicles negatively regulate $\mathrm{NK}$ cell function by a mechanism involving TGF- $\beta$ and miR23a transfer. OncoImmunology 5: e1062968, 2015.

23. Li L, Li C, Wang S, Wang Z, Jiang J, Wang W, Li X, Chen J, Liu K, Li C, et al: Exosomes derived from hypoxic oral squamous cell carcinoma cells deliver miR-21 to normoxic cells to elicit a prometastatic phenotype. Cancer Res 76: 1770-1780, 2016.

24. Ying $X$, Wu Q, Wu $X$, Zhu Q, Wang $X$, Jiang L, Chen $X$ and Wang $X$ : Epithelial ovarian cancer-secreted exosomal miR-222-3p induces polarization of tumor-associated macrophages. Oncotarget 7: 43076-43087, 2016.
25. Montecalvo A, Larregina AT, Shufesky WJ, Stolz DB, Sullivan ML, Karlsson JM, Baty CJ, Gibson GA, Erdos G, Wang Z, et al: Mechanism of transfer of functional microRNAs between mouse dendritic cells via exosomes. Blood 119: 756-766, 2012.

26. Yang HW, Liu GH, Liu YQ, Zhao HC, Yang Z, Zhao CL, Zhang XF and Ye H: Over-expression of microRNA-940 promotes cell proliferation by targeting GSK $3 \beta$ and sFRP1 in human pancreatic carcinoma. Biomed Pharmacother 83: 593-601, 2016.

27. Liu X, Kwong A, Sihoe A and Chu KM: Plasma miR-940 may serve as a novel biomarker for gastric cancer. Tumour Biol 37: 3589-3597, 2016.

28. Su S, Liu Q, Chen J, Chen J, Chen F, He C, Huang D, Wu W, Lin L, Huang W, et al: A positive feedback loop between mesenchymallike cancer cells and macrophages is essential to breast cancer metastasis. Cancer Cell 25: 605-620, 2014.

29. Desir S, Dickson EL, Vogel RI, Thayanithy V, Wong P, Teoh D, Geller MA, Steer CJ, Subramanian S and Lou E: Tunneling nanotube formation is stimulated by hypoxia in ovarian cancer cells. Oncotarget 7: 43150-43161, 2016.

30. Leblond MM, Gérault AN, Corroyer-Dulmont A, MacKenzie ET, Petit E, Bernaudin M and Valable S: Hypoxia induces macrophage polarization and re-education toward an M2 phenotype in U87 and U251 glioblastoma models. OncoImmunology 5: e1056442, 2015

31. Zhang J, Cao J, Ma S, Dong R, Meng W, Ying M, Weng Q, Chen Z, Ma J, Fang Q, et al: Tumor hypoxia enhances non-small cell lung cancer metastasis by selectively promoting macrophage M2 polarization through the activation of ERK signaling. Oncotarget 5: 9664-9677, 2014.

32. Saini U, Naidu S, ElNaggar AC, Bid HK, Wallbillich JJ, Bixel K, Bolyard C, Suarez AA, Kaur B, Kuppusamy P, et al: Elevated STAT3 expression in ovarian cancer ascites promotes invasion and metastasis: A potential therapeutic target. Oncogene 36: 168-181, 2017

33. Wu Y, Deng W, McGinley EC and Klinke DJ II: Melanoma exosomes deliver a complex biological payload that upregulates PTPN11 to suppress T lymphocyte function. Pigment Cell Melanoma Res 30: 203-218, 2017.

34. Wu L, Zhang X, Zhang B, Shi H, Yuan X, Sun Y, Pan Z, Qian H and $\mathrm{Xu} \mathrm{W}$ : Exosomes derived from gastric cancer cells activate $\mathrm{NF}-\kappa \mathrm{B}$ pathway in macrophages to promote cancer progression. Tumour Biol 37: 12169-12180, 2016.

35. Beatson R, Tajadura-Ortega V, Achkova D, Picco G, Tsourouktsoglou TD, Klausing S, Hillier M, Maher J, Noll T, Crocker PR, et al: The mucin MUC1 modulates the tumor immunological microenvironment throughengagement of the lectin Siglec-9. Nat Immunol 17: 1273-1281, 2016. 